\title{
Construcción de un marco teórico para las ciencias de la gestión
}

\section{Construction of a theoretical framework for Management Sciences}

Julio Enrique Foliaco-Gamboa ${ }^{\mathrm{a}^{*}}$, Mayra Esperanza Foliaco-Albino ${ }^{\mathrm{b}}$

${ }^{a}$ Especialista en Sistema de Gestión de la Calidad, jefoliacog@unal.edu.co, orcid.org/0000-0002-1186-2701, Universidad Nacional de Colombia, Bogotá, Colombia.

${ }^{b}$ Maestria en Marketing, mayfol@hotmail.com,orcid.org/0000-0002-2785-4503, Fundación de estudios Superiores FESC, Cúcuta, Colombia.

Cómo citar: J.E. Foliaco-Gamboa y M.E. Foliaco-Albino, "Construcción de un marco teórico para las ciencias de la gestión", Respuestas, vol. 23, no. S1, pp. 86-96, 2018. https://doi.org/10.22463/0122820X.1523

Recibido: Enero 25, 2018; Aprobado: Junio 30, 2018

\section{RESUMEN}

Palabras Clave:

Auto - organización

Ciencias de la complejidad

Ciencias de la gestión

Estudios críticos de gestión

Pensamiento complejo

Post - estructuralismo

Sistemas adaptativos complejos

\section{Keywords:}

Auto - organization

Science of complexity

Management science

Critical studies of management

Complex thinking

Post - structuralism

Complex adaptive systems
El pensamiento complejo, las ciencias de la complejidad y la auto-organización son los nuevos referentes teóricos para las ciencias de la gestión en el marco de la ciencia contemporánea. Instituciones internacionales como la Escuela de Humanismo y Gestión (HEM) en Canadá, la Escuela de Economía de Londres (LSE), y el Instituto Santa Fe (SFI) en USA, así como las corrientes de pensamiento de la Escuela de Frankfurt liderada por Max Horkheimer y la Escuela Postmodernista de Michael Foucault, han enriquecido la construcción de una epistemología para las ciencias de la gestión. Los propósitos específicos son: 1. Analizar las teorías y críticas recibidas por la gestión clásica y lineal provenientes del pensamiento post - estructuralista; 2 . Destacar las teorías y la praxis de esta nueva corriente teórica para la gestión; 3. Comparar los conceptos de gestión post-estructuralistas con la evolución de las ciencias de la gestión; y 4. Identificar ámbitos de acción específicos donde se generen cambios estructurales en los programas académicos de las ciencias de la gestión.

\section{Introducción}

La administración como disciplina surge a finales del siglo XIX para organizar el alto volumen de producción generado por la Revolución Industrial dos siglos antes. La gestión empresarial centrada en la llave propietarios - gerentes ha mantenido el poder sobre los medios de producción con base en la estandarización de procesos y en la maximización de la rentabilidad del capital invertido, en otras palabras, las ciencias de la gestión (CG), han sido orientadas por el pensamiento cartesiano y estructuralista desde las décadas finales del siglo XIX hasta la actualidad. Un apreciable volumen de investigaciones y estudios críticos relativos a la instrumentalización del trabajador y la inequidad en la distribución de beneficios entre el capital y el trabajo surgieron en la década de 1980. El deterioro de la calidad de vida y el medio ambiente se atribuye a las ciencias de la gestión (CG) por parte de reconocidos críticos. Asimismo, la ciencia moderna y cartesiana, tan influyente en el mecanicismo y el estructuralismo administrativo, está siendo superada por corrientes de pensamiento complejas y contemporáneas.

En la actualidad se desarrollan investigaciones y aplicaciones para promover estrategias de gestión apoyadas en las ciencias de la complejidad (CC), el pensamiento complejo y la auto-organización asociada con la biología del pensamiento. Con esta reciente tendencia convergen los Estudios Críticos de Gestión (CMS, Critical Management Studies por sus siglas en inglés), inspirados en escritos anglosajones como el Trabajo y Capital Monopolista de Braverman y la obra Vigilar y Castigar de Foucault. Los CMS denuncian la degradación, el excesivo control y la instrumentalización del individuo en las organizaciones. Simultáneamente se crean líneas de investigación en London School of Economics (LSE) de Inglaterra, institución considerada pionera de los estudios críticos desde la década de 1980. Otros artífices de estas críticas se encuentran en la Escuela de Frankfurt en Alemania, encabezados por Jürgen Habermas y su teoría social para el análisis del capitalismo avanzado. En la Escuela Canadiense de Humanismo y Gestión (HEM) liderada por Omar Aktouf y enfocada a rechazar las prácticas inhumanas en las organizaciones. En Colombia se crea la Escuela del Nuevo Pensamiento Administrativo en la Universidad del Valle con el respaldo de la HEM, orientada a recobrar la dignidad del individuo y a la incorporación de nuevas epistemologías para la formación en gestión.

Con respecto a la investigación y la praxis de esta nueva corriente surgen los Sistemas Adaptativos Complejos (CAS,

*Autor de correspondencia.

E-mail: jefoliacog@unal.edu.co (Julio Foliaco Gamboa).

cc) (i) (s) $\odot$ Peer review is the responsibility of the Universidad Francisco de Paula Santander. (c) 1 ay 20 This is an article under the license CC BY-NC-ND 
Complex Adaptative Systems por sus siglas en inglés) liderados por el Instituto Santa Fe (SFI) con sede en Nuevo México, Estados Unidos, fundado en 1984. Investigadores como Kauffman, Gell - Mann, Hollman, Stacey, Anderson y otros, crean metodologías para la auto-organización en el marco de los sistemas complejos. Los CAS se han convertido en una respuesta a las críticas sobre la excesiva abstracción y la poca praxis ofrecida por los CMS.

Por su parte, las ciencias de la complejidad (CC) explican sistemas universales con base en fenómenos naturales y artificiales, asocian la gestión con la naturaleza cambiante de estos fenómenos y se opone a los procesos de estandarización, al mundo de certezas y al condicionamiento cuantitativo promovido por la corriente clásica y estructuralista de la gestión. Para las CC, la gestión es per se una complejidad caracterizada por la disipación de estructuras, las analogías, las bifurcaciones, las emergencias y el carácter impredecible de las estrategias [1]. Por su parte, el concepto de auto - organización como conjunto de interacciones cotidianas entre los integrantes de un sistema, se centra en la autonomía natural caracterizada por la regeneración y la corrección del desorden, propio de los sistemas orgánicos [2]. Y tiene ocurrencia en los sistemas biológicos como fenómenos vitales, en una dinámica compleja de relaciones continuas entre los individuos y el medio [3].

En el estudio "Dinámicas identitarias y espacios públicos” [4], los seres humanos como organizaciones vitales se desorganizan y reorganizan en forma compleja y en una dialógica permanente de orden - desorden que conduce a estados transitorios auto - organizados. El mayor avance de esta idea se encuentra en los escritos sobre el pensamiento complejo [5], quien asocia la auto - organización con un proceso auto-producido por el insumo de la información, comparándolo con la autopoiésis del estudio [6]. Así, un proceso auto - organizado asociado con un proceso auto-poiético se provee de información interna tal como el proceso molecular generado en forma autónoma e independiente. También, recibe información contextual y estructural semejante a la información genética inscrita en el ADN [7]. En esta perspectiva, se propone que las iniciativas y la descentralización de decisiones en la organización empresarial sean guiadas por una gestión auto - organizada.

Adicionalmente, la construcción de planes desde la cúpula organizacional sin tener en cuenta el conocimiento disperso y el orden espontáneo, genera una "arrogancia fatal" para la planificación porque los directivos sucumben a la creencia de disponer de toda la información existente [8]. Por su parte Von Foerster [9] identifica la flexibilidad de adaptación, el control limitado y la construcción de decisiones compartidas como las cualidades de un proceso auto - organizado. Para Crozier [10], sí bien la gestión lineal y jerarquizada obtiene cierta racionalización de actividades colectivas, acarrea el riesgo de la estandarización y el sometimiento de los individuos.
En síntesis, el proceso auto-organizado se convierte en el eje principal de una nueva epistemología para la gestión, amparada por las ciencias de la complejidad, el pensamiento complejo, las corrientes de los CMS y los CAS. Igualmente, es una respuesta a las fuertes críticas recibidas por la gestión clásica, originadas por sus impactos negativos en la instrumentalización del individuo, la burocratización, el control centralizado, el agotamiento de recursos naturales, la inequidad social y los conflictos sociales. Invocando una expresión aristotélica, las ciencias de la gestión no han contribuido al "bien - vivir" de la comunidad.

\section{Nueva génesis conceptual para las ciencias de la gestión}

Esta construcción comienza con la comparación entre el pensamiento clásico administrativo y la nueva corriente compleja del pensamiento. La primera, caracterizada por su énfasis en la división del trabajo, la especialización, la jerarquía, los resultados, el estructuralismo y el antropocentrismo. La segunda, fundamentada en las emergencias, las interrelaciones, la disipación, el control descentralizado, la robustez y la creatividad con base en verdades analógicas. El propósito es confrontar el sistema de gestión lineal y el sistema complejo cimentado en la auto-organización, descrito en la siguiente manera (ver Tabla I).

Tabla I. Comparación entre el sistema de gestión lineal y el sistema de auto - organización

\begin{tabular}{|c|c|}
\hline Gestión con linealidad & Gestión con auto-organización \\
\hline $\begin{array}{c}\text { 1. Información: apoyada en manuales de } \\
\text { funciones y estándares de producción }\end{array}$ & Información: apoyada en la cibernética y los sistemas \\
\hline $\begin{array}{c}\text { 2. Instrumentos de operación: planes y y } \\
\text { programas pre - diseñados. Uso de la } \\
\text { lógica clásica }\end{array}$ & $\begin{array}{c}\text { Instrumentos de operación: emergencias/acuerdos como } \\
\text { referentes de la dinámica organizacional. Aplicación de } \\
\text { las lógicas no clásicas: abductiva, difusa. }\end{array}$ \\
\hline $\begin{array}{c}\text { 3. Procesos de decisión: centralizados, } \\
\text { burocráticos y estandarizados. Poca } \\
\text { autonomía. }\end{array}$ & $\begin{array}{c}\text { Procesos de decisión: descentralizados por consensos, } \\
\text { disensos y grupos. Con autonomía y acuerdos } \\
\text { colectivos contingentes. Adyacentes posibles. }\end{array}$ \\
\hline $\begin{array}{c}\text { 4. Contexto: Baja preocupación por las } \\
\text { personas y el medio ambiente }\end{array}$ & $\begin{array}{c}\text { Contexto: Beneficios sociales para el ambiente y las } \\
\text { personas interesadas en la organización. }\end{array}$ \\
\hline
\end{tabular}

Fuente: Introducción a la Teoría General de la Administración [11]

La idea primordial es aportar las bases teóricas para encontrar la identidad propia de las ciencias de la gestión con base en teorías comprometidas con el desarrollo antropo - social y la complejidad natural y organizacional. Con la orientación clara de cambiar el estructuralismo disyuntor de realidades o reductor de complejidades, predominante en las explicaciones fenoménicas de la ciencia moderna o cartesiana [12]. A cambio, incluir las corrientes científicas contemporáneas del pensamiento complejo, las ciencias complejas, la auto-organización, los CMS y los CAS para dar respuesta a las restricciones de la corriente lineal y a los problemas de la vida cotidiana generados por la gestión como medio de sobrevivencia [13].

Por su parte, las CC comportan actividades auto-organizadas y emergen para conocer fenómenos imprevistos e indescifrables por las teorías apoyadas en la causalidad lineal. Las críticas a 
las ciencias de la gestión provienen de los centros e institutos dedicados al estudio de los sistemas complejos y datan de los primeros años de la década de 1980. Maldonado en un intento de establecer una diferencia entre la corriente lineal y la corriente compleja, define así las CC:

Estas ciencias son un conjunto de disciplinas, teorías, enfoques, lenguajes y métodos qué se dedican al estudio, por decirlo de manera fuerte y por vía de contraste, de todo aquello que no estudia la ciencia normal. Notablemente, se trata de ciencias y disciplinas que incluyen teorías como el caos, la geometría de fractales, las catástrofes, la termodinámica y la ciencia de las redes complejas [14].

Como complemento a esta conceptualización, El estudio de Morin "El Método II: La Vida de la Vida" [7] asocia la auto organización con la autopoiésis de [6], porque implica un proceso sostenible en el tiempo con manejo autónomo de información interna e influenciado por estímulos ambientales. En este caso, el prefijo auto es explicado por la presencia de estímulos externos decantados por el individuo en función de su propia vitalidad y autonomía. La autopoiésis es la auto-organización de lo vivo a partir del auto-mantenimiento de las células vivas capaces de reproducirse y mantenerse [15]. Analógicamente, el ser humano es una máquina que se organiza por sí misma en forma constante, con una autonomía vital propiciada por un entorno, un campo fenoménico y un campo genético en función de su propia regeneración individual [7].

En el marco de la gestión, el concepto funcional de auto organización traza un nuevo orden a partir de las interacciones de los componentes de un sistema [16]. También, se entiende como un conjunto de interacciones entre miembros de diferentes niveles para generar sus propias estructuras. La información proveniente de las interacciones es propia del sistema, no impuesta externamente, lo cual constituye la robustez, la originalidad y la creatividad de un proceso auto - organizado [17]. Por consiguiente, la cotidianidad de las interacciones entre los miembros de la organización confiere a las estructuras un carácter disipativo producido por el intercambio de información con el entorno y su previa decantación interna, cambiando patrones actuales y facilitando la emergencia de nuevos modelos [18]. No obstante, la auto organización sólo ocurre en sistemas abiertos e importadores de energía [19] o información del entorno y logran estados de inestabilidad limitada [20].

Este análisis encamina a un modelo auto-organizado, apoyado en principios de complejidad para la búsqueda de una identidad propia de las ciencias de la gestión, e implica una respuesta a las críticas hechas por el estudio [21] sobre el sometimiento de la gestión a las epistemologías de otras disciplinas, la sobreprotección al capital y la carencia de principios originados al interior del saber y el quehacer administrativo. De acuerdo con trabajo "At Home in the Universe: The Search for the Laws of Self-Organization and Complexity" [16], el devenir de un nuevo orden requiere incorporar conceptos esenciales como las emergencias, las interrelaciones, la co-evolución, la flexibilidad y la robustez. El estudio "Complex adaptative systems" [20] agrega la inestabilidad limitada. Najmanovich [22] adiciona el contexto social, natural y económico. En consecuencia, los sistemas deben tener como característica fundamental una alta sensibilidad a las reacciones individuales y a las emergencias impulsoras de la criticalidad auto-organizadora y generadora de conceptos como las bifurcaciones, las estructuras disipativas, las asimetrías, las analogías y la relatividad [23]. En conjunto, esta construcción teórica incluye conceptos de la biología, la física y la sociología, en una simbiosis evolutiva entre la corriente de pensamiento lineal y la corriente compleja, considerando la auto-organización como concepto esencial de las ciencias de la gestión (Figura 1).

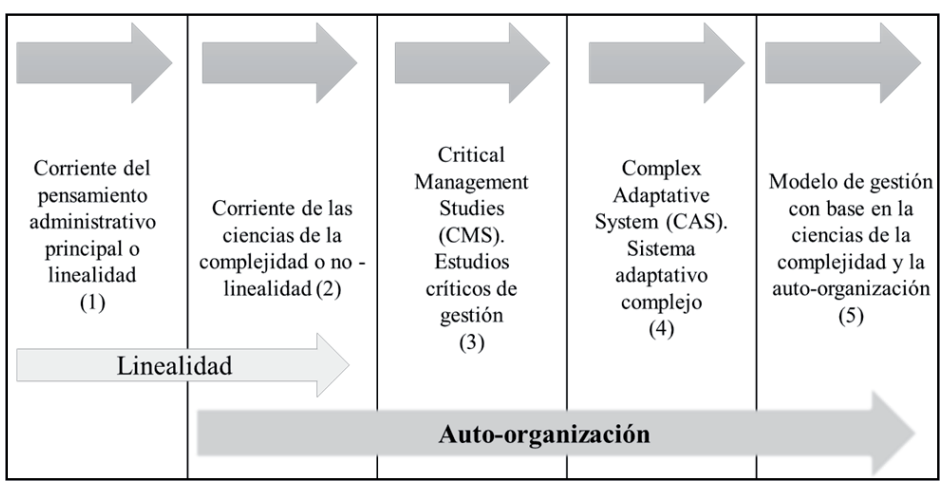

Figura 1. Proceso de construcción de las ciencias de la gestión con base en la auto organización

La columna inicial de la Figura 1 condensa el estado actual de las ciencias de la gestión con su carácter lineal, analítico, mecanicista y determinista en la instrumentalización del trabajador. La segunda columna designa las ciencias de la complejidad reconocidas por la comunidad científica como "ciencia normal" [24], atribución adquirida por su coherencia teórica y su praxis en favor de la sociedad. Los fuertes cuestionamientos hechos por la corriente de los CMS se convierten en factor significativo en la propuesta de innovaciones substanciales para las ciencias de la gestión, señalado en la tercera columna. La compilación de investigaciones y nueva terminología generada por los CAS tienen una nomenclatura propia surgida de los estudios y las aplicaciones de los sistemas complejos, se aluden en la cuarta columna. Este desarollo conduce a un nuevo marco para las ciencias de la gestión fundamentado en la complejidad y la auto-organización, suscrito en la columna cinco. Es oportuno distinguir dos corrientes dentro el pensamiento complejo: la primera representada por la teoría general de sistemas, inspirada en la necesidad de una ciencia interdisciplinaria para servir de soporte informativo a las demás ciencias [25]. Esta corriente se complementa con la cibernética, los métodos matemáticos, los sistemas estocásticos y se considera adaptativa por su dependencia de factores externos. La segunda corriente de la complejidad se caracteriza por dinámicas inestables y de causalidad múltiple, propias de sistemas 
auto-organizados como el cerebro, la biosfera y las organizaciones empresariales [26]. De esta forma se reconoce que las organizaciones detentan ciclos intrínsecos no lineales por la interacción de las personas sin distinción alguna [27].

En general, los sistemas complejos intercambian materia, energía e información como conjunto de elementos en interacción, mientras que un sistema simple genera resultados no deducibles del agregado de sus partes. Así, las neuronas del cerebro son partes en interacción que no pueden ser consideradas separadamente o en forma yuxtapuesta y tampoco son reductibles a sus componentes, ni explicadas por sus propiedades emergentes [28]. Además, la complejidad recibe aportes de la interdisciplinariedad para la exploración de diferentes caminos de aprendizaje y generación de estructuras disipativas sin control centralizado. Incluye intercambio de métodos, combinación de metas, aplicación de instrumentos y verdades analógicas, sin recurrir al sistema tradicional jerarquizado [29]. Estos factores disipativos y analógicos no se ajustan a métodos lineales o singulares, al contrario se orientan hacia la complejidad creciente caracterizada por la aplicación de diferentes dominios disciplinarios [30].

En síntesis, se propone un modelo conceptual orientado por las $\mathrm{CC}$, el PC, los CAS, los CMS, con el fundamento de auto organización. Esta construcción está encauzada por las teorías emergentes de la complejidad, los estudios críticos de las ciencias de la gestión, la interdisciplinariedad y el significativo volumen de análisis y praxis fomentado por los sistemas adaptativos complejos.

\section{Hacia un nuevo modelo para las ciencias de la gestión}

Los siguientes escritos son cruciales en la construcción de un nuevo marco teórico para las ciencias de la gestión: La Crítica a la razón instrumental de Horkheimer [31], editada en 1937; El Trabajo y capital monopolista de Braverman [32]; Vigilar y castigar de Foucault [33]; y la Teoría de la acción comunicativa de Habermas [34]. Estas obras coinciden en cuestionar el excesivo apego al libre mercado, la instrumentalización del trabajo, el intensivo control, la baja participación y la orientación hacia la sociedad disciplinaria de la gestión lineal. En la Tabla II se describen los pilares de la nueva construcción compleja de la gestión: el pensamiento complejo, los estudios críticos y las ciencias de la gestión, los cuales convergen con base en el factor esencial de auto - organización.
Tabla II. Construcción de un nuevo marco para las ciencias de la gestión

\begin{tabular}{|c|c|c|}
\hline Enfoques y autores & Trabajos seminales & Aportes principales \\
\hline $\begin{array}{c}\text { 1. Sistemas Adaptativos Complejos } \\
\text { (CAS) } \\
\text { Holland, Stacey, Mitleton, Kauffman, } \\
\text { E. Bohorquez. Instituto Santa Fé } \\
\text { (SFI). London School of Economics } \\
\text { (LSE) }\end{array}$ & $\begin{array}{c}\text { Los sistemas complejos y } \\
\text { de complejidad creciente. } \\
\text { Los modelos de auto } \\
\text { organización empresarial } \\
\text { Adaptación igual a } \\
\text { evolución }\end{array}$ & $\begin{array}{l}\text { La criticalidad inherente a los sistemas } \\
\text { naturales y artificiales. } \\
\text {-La emergencia, la evolución y la auto } \\
\text { organización = CAS = Organización } \\
\text { Empresarial: orden - desorden - auto- } \\
\text { organización. } \\
\text { - Los CAS: interacciones vs. acciones } \\
\text { individuales. }\end{array}$ \\
\hline $\begin{array}{l}\text { 2. Estudios Críticos de Gestión } \\
\text { (CMS). } \\
\text { Le Moigne, Braverman, Aktouf, } \\
\text { Stiglitz, García. }\end{array}$ & $\begin{array}{l}\text { Trabajo y capital } \\
\text { monopolista; } \\
\text { - Incoherencia } \\
\text { epistemológica de las } \\
\text { ciencias de la gestión; } \\
\text { - La crisis de los MBA. }\end{array}$ & $\begin{array}{l}\text { - La búsqueda de legitimidad } \\
\text { epistemológica de las ciencias de la } \\
\text { gestión. } \\
\text { - Critica a la reducción de niveles de } \\
\text { cualificación y el control social del } \\
\text { trabajo. Capital económico prioridad } \\
\text { frente al capital humano }\end{array}$ \\
\hline $\begin{array}{l}\text { 3. Pensamiento Complejo Edgar } \\
\text { Morin, Maturana, D.Namajnovich, } \\
\text { Prigonine Universidades: Militar, } \\
\text { Rosario, ICESI. Weiner, Bertallanfy, } \\
\text { Von Neuman }\end{array}$ & $\begin{array}{c}\text {-Principios de la } \\
\text { complejidad: la dialógica, } \\
\text { la recursión y la } \\
\text { hologramía. } \\
\text { La complejidad y la } \\
\text { autopoiésis El orden y el } \\
\text { desorden La cibernética y } \\
\text { los sistemas como } \\
\text { referentes }\end{array}$ & $\begin{array}{l}\text { - La recursión, la dialógica y la } \\
\text { contextualización como esenciales en una } \\
\text { nueva forma de investiga r/pensar. } \\
\text { - La autopoiésis: acople individuo - } \\
\text { entorno. Autopoiésis es auto generación. } \\
\text { El lenguaje es biológico y simbólico. }\end{array}$ \\
\hline
\end{tabular}

El Instituto Santa Fé (SFI) es la institución abanderada de la investigación sobre los sistemas adaptativos complejos (CAS). Es la promotora de un nuevo perfil académico para la gestión a partir de las aplicaciones del pensamiento complejo y las ciencias de la complejidad [23]. En particular, los CAS brindan nuevos meta-conceptos: emergencias, interrelaciones, evolución-co-evolución, flexibilidad, devenir, robustez, y auto organización como meta-conceptos [35], [36]. En forma correlativa, las organizaciones empresariales como sistemas complejos están asociadas con los comportamientos autopoiéticos de los seres vivos, sus desempeños culturales y el desarrollo de los sistemas sociales [6]. Las organizaciones se consideran sistemas vivos porque funcionan con el resguardo de los seres humanos y en el marco de los principios de auto - organización [9]. Además, la complejidad enfatiza en la integración entre los individuos y las organizaciones con base en componentes autónomos que interactúan y modifican permanentemente los sistemas [37]. Por consiguiente, las ciencias de la complejidad, la teoría general de sistemas [38], la cibernética [39] y los principios de la complejidad [5] son pilares conceptuales de un sistema auto - organizado.

Las ciencias de la complejidad (CC), reconocidas por la comunidad científica mundial, laureadas con un Nobel y robustecidas con investigaciones teórico - prácticas, en las últimas cuatro décadas, son cruciales en la construcción de un nuevo marco teórico para las ciencias de la gestión. Entre las CC se identifican la termodinámica del no equilibrio [19]; la teoría del caos [40] -muy conocida por la parábola del "efecto mariposa"-; la geometría de fractales [41]; la teoría de la catástrofe [42]; y la teoría de redes [43]. El contorno analógico de estas teorías ha conformado el campo de investigación de mayor crecimiento en el estudio de las organizaciones como sistemas no lineales en las últimas cuatro décadas [44]. La construcción de esta nueva perspectiva con base en las nuevas ciencias de la gestión (NCG) se resume en el siguiente algorit- 
mo simple (1):

$$
N C G=C M S+C A S+C C+P C
$$

En particular, en el marco de la corriente contemporánea de pensamiento y dentro de las escuelas anglosajonas, francesas, norteamericanas y latinoamericanas se consolida la teoría y la praxis del pensamiento complejo desde la segunda mitad del siglo XX. Es un pensamiento fundamentado en la criticalidad o permanente variabilidad y la evolución vigorosa de los fenómenos físicos, humanos, sociales y económicos. A su vez, este concepto se convierte en el cimiento de las ciencias de la complejidad, reconocidas por la comunidad científica, aunque aún resistidas por académicos de la corriente principal [14]. Se trata de un universo de complejidad creciente con emergencias, turbulencias, fluctuaciones, incertidumbres y auto - organización [16], [36], [45], [46]. Entra la era de las verdades temporales y espaciales que confrontan el absolutismo, la dualidad medioeval, el renacentismo, la lógica cartesiana y el rechazo a la verdad sin evidencia plena [47]. Las ciencias de la gestión y todas las ciencias se ven avocadas a enfrentar sus verdades actuales, modernas y pre-modernas con las nuevas realidades del pensamiento contemporáneo, impregnado por las dinámicas de la complejidad, el orden - desorden y el determinismo contingencia.

\section{Las críticas convergentes a las ciencias de la gestión}

Resulta paradójico que las críticas a las ciencias de la gestión se originen en las mismas escuelas o facultades de administración y gestión con notables aportes de autores de otras disciplinas. En el artículo La incoherencia epistemológica de las ciencias de la gestión se cuestiona el excesivo positivismo y dependencia epistemológica de otras disciplinas [21]. Igualmente, se reitera la crítica en la obra The New Science of Management Decision, sobre la excesiva fascinación de la gestión por las técnicas matemáticas, particularmente por las finanzas, con la consecuente desatención a la observación empírica del comportamiento humano y sus complejidades [48]. En el mismo artículo, Le Moigne discrepa de los lineamientos cartesianos que fomentan el determinismo, el reduccionismo, el simplismo y además recomienda a los académicos de la gestión, conocer obras como El nuevo espíritu científico de Brachelard y La Tectología de Bodganov, difundidas en 1937 y 1920 respectivamente. Ambos autores propusieron la unificación de las ciencias físicas, biológicas y sociales e inspiraron los enfoques sistémicos de Wiener y Bertalanffy, surgidos en la mitad de siglo XX.

Para Horkheimer, citado por [49], no pensar en las cosas del momento a cambio de cumplir normas y procedimientos impli- ca desconocer el carácter creativo de la razón y negar su condición esencial de pensar. En el ámbito del pensamiento clásico, el intento de instrumentalizar la mente de los trabajadores origina una actitud despectiva frente a las normas y los objetivos impuestos por la organización. Esta crítica es coherente con el reconocimiento a la innata necesidad del individuo de auto-organizarse, robustecerse y ser creativo para enfrentar las emergencias y las novedades cotidianas. En este marco, las ideas de Max Horkheimer (1875 - 1973), presentan profundas similitudes con la Escuela de Frankfurt y los CAS en relación con la alienación, la reificación del individuo, la cultura del mercado y las supuestas coincidencias de intereses entre el capital y el trabajo. Adicionalmente, Braverman [32] cuestiona la alienación de sectores cada vez más amplios de la población trabajadora dentro de la empresa y en su vida social como resultado de la mecanización, la estandarización y la burocratización. También critica la gerencia por la degradación de la cualificación y los puestos de trabajo. Por su parte, Foucault [33] compara la gestión empresarial con el panóptico y la sociedad disciplinaria, mientras asocia el accionar carcelario con la organización lineal y atribuye al control la depredación de la subjetividad y la creatividad de los individuos. En esta misma perspectiva, Fernández [50] retoma las críticas al instrumentalismo, la alienación y reificación del individuo bajo la cultura del mercado hecha por la Escuela de Frankfurt.

Estas críticas a las ciencias de la gestión se inician en la Escuela de Economía de Londres (LSE) en la década de los 80 y se enfocan en las prácticas de inhumanidad organizacional [51]. Por su parte, Juan Saavedra [52], egresado de la Universidad Nacional de Colombia (UNAL), en su artículo El lado oscuro de la gestión, analiza el estado del arte de los CMS y registra el avance de esta nueva corriente del pensamiento como una contribución al desarrollo y renovación de la disciplina administrativa. Saavedra exalta el trabajo hecho por los laboratorios de investigación en Estrategia, estructura y decisión de la Universidad Nacional, liderados por Ricardo Romero y el trabajo del grupo Perdurabilidad empresarial de la Universidad del Rosario, orientado por Carlos Eduardo Maldonado. Asimismo, hace alusión a importantes trabajos sobre las ciencias de la complejidad realizados por la Universidad Nueva Granada, La Salle, ICESI de Cali, Distrital y El Rosario. Para Saavedra, los CMS responden a la necesidad de construir una nueva teoría para la gestión que interprete los cuestionamientos sobre la racionalidad instrumental predominante en las organizaciones contemporáneas. Se considera que existe un volumen crítico suficiente de insumos académicos para consolidar una nueva escuela para el desarrollo de los CMS e implementar la tendencia compleja y auto-organizadora de las ciencias de la gestión en Colombia.

Según el profesor Fernando Cruz [53], las universidades requieren renovar sus currículos de gestión, tanto de pregrado como de postgrado, con contenidos que incluyan la nueva corriente de los CMS. Cruz, presenta evidencias sobre el éxito empírico de esta renovación, ya iniciada en la Universidad del 
Valle, haciendo referencia específica al caso de la empresa PAVCO en el municipio de Chechene (Colombia), donde la aplicación de preceptos humanísticos contribuye con la consolidación de organizaciones en torno al concepto de dignidad, aplicado en un contexto de gran diversidad étnica y cultural propias de la región. Precisa el profesor Cruz, que, si bien la definición de dignidad es compleja, las acciones contra la dignidad sí pueden ser identificadas para establecer qué es dignidad por las acciones que la niegan o la desconocen.

Igualmente, los CMS representan una línea de investigación social fundamentada en denuncias sobre el desconocimiento cultural autóctono y las injusticias de los sistemas centrados en el capital y el antropocentrismo. Es una corriente arraigada en el pensamiento complejo y en la confrontación de inequidades sociales y económicas. Los siguientes autores cibernéticos, sistémicos y epistemólogos son considerados pioneros de la corriente compleja en la órbita de la ciencia contemporánea: Gaston Brachelart, Frederick Hiedegger, Thomás Kuhn, Jean-Louis Le Moigne, Frederick Hegel, Frederick Nietzsche, John von Neumann, Norbert Wiener, Ludwig Bertalanffy, Edward Deming, Alfred Chandler y Edgar Morin, entre otros. La apuesta asumida por estos autores implica una nueva forma de pensar multidisciplinaria y compleja. Incluso, reconocidos filósofos como Bunge [54] expresan una mayor preocupación por la ética, la sociología, la complejidad y la política a instancias de la metafísica. Para Cruz [51], el pensamiento humano, en el escenario de la historia, ha evolucionado de lo mágico y mítico, propio de las sociedades ancestrales, a lo lógico, racional y científico de la nueva corriente del pensamiento complejo y analógico.

Las críticas apuntan al excesivo énfasis en la comunicación y la cultura por parte de los CMS, mientras olvidan que el trabajo y las organizaciones deben ser los objetos principales de estudio. Aunque, se reconoce la existencia de un amplio volumen de ensayos y escritos, la crítica más generalizada a los CMS es su falta de carácter empírico para apoyar el discurso teórico sobre la realidad social que se está cuestionando. Sin embargo, todos los contradictores coinciden en la necesidad de nuevas propuestas sobre la teoría organizacional y de un mayor pragmatismo que evite el aburguesamiento intelectual de las actuales generaciones. Específicamente, es necesario superar la simple formación técnica en mercadeo, finanzas y administración de personal, así como la adhesión a principios de obediencia y competitividad, inspirados en los dogmas de los gurús del management [50]. Surge el interés por la preocupación natural de los seres humanos por los acontecimientos y la situación socio-económica de sus congéneres, en la denominada biología cultural asociada con la biología del lenguaje [55].

\section{Aportes de los sistemas complejos a las ciencias de la gestión}

Las investigaciones y aplicaciones sobre los sistemas comple- jos realizadas por el SFI son una reacción explícita a las críticas provenientes de la corriente de los CMS. Asimismo, son un aporte metodológico para el desarrollo de los estudios enmarcados en la complejidad. Es preciso analizar los principios, las características y los resultados de estos estudios para mejor comprensión de la auto - organización y su despliegue conceptual en la gestión. El SFI con los trabajos liderados por Stuart Kauffman, Gell - Mann y John Holland, entre otros, es la institución pionera en la praxis de los sistemas adaptativos complejos a la gestión. Las revistas académicas de gran prestigio como Organization Science, The Learning Organization, Human Systems Management, Complexity and Organization, son los principales medios difusores de estos estudios. La expresión "adaptativos", utilizada en los CAS tiene una connotación biológica de evolución y no de simple acomodación y su mayor logro es establecer que la gestión depende más de interacciones de los trabajadores que de las decisiones centralizadas de los directivos. Por tanto, el éxito de la gestión está supeditado a los eventos generados por la dialógica permanente entre el orden y el desorden, así como a la evolución, co-evolución y aprendizaje mutuos provocados por las interacciones laborales. Los cambios conceptuales aportados por los CAS se señalan en la siguiente comparación entre los conceptos de la corriente lineal y la corriente compleja de la gestión (Tabla III):

Tabla III. Comparación entre los conceptos utilizados por la corriente lineal y la corriente compleja

\begin{tabular}{|c|c|}
\hline $\begin{array}{c}\text { Corriente principal de la } \\
\text { Administración: lineal o clásica }\end{array}$ & $\begin{array}{c}\text { Auto-gestión Empresarial: no } \\
\text { lineal o compleja }\end{array}$ \\
\hline Causa principal Causalidad lineal & $\begin{array}{c}\text { Confluencia de causas Causalidad } \\
\text { múltiple }\end{array}$ \\
\hline El orden & El orden y el desorden \\
\hline Estructura formal & Estructura disipativa \\
\hline $\begin{array}{c}\text { Problemas } \\
\text { Dualidad }\end{array}$ & $\begin{array}{c}\text { Emergencias/Novedades } \\
\text { Conjunciones - convergencias } \\
\text { Inestabilidad limitada }\end{array}$ \\
\hline $\begin{array}{c}\text { Alto grado de estabilidad } \\
\text { Agregado de acciones individuales }\end{array}$ & $\begin{array}{c}\text { Interacciones- equipos Agregado de } \\
\text { interacciones colectivas }\end{array}$ \\
\hline $\begin{array}{c}\text { Simplicidad } \\
\text { Retroalimentación }\end{array}$ & $\begin{array}{c}\text { Simplicidad y complejidad Recursión } \\
\text { Decisiones centralizadas }\end{array}$ \\
\hline Control centralizado & Decisiones descentralizadas \\
\hline Resultados programados & Criticalidad, Innovación y creatividad \\
\hline
\end{tabular}

Fuente: Complex adaptative systems [20], The science of complexity: An alternative perspective for strategic changing processes [27], Hidden order: How adaptation builds complexity [37].

En la Tabla III, se compendian los estudios de [20], [27], [37] sobre los conceptos necesarios para crear un sistema complejo de gestión. Estos postulados conllevan instrumentos para la coexistencia de la estabilidad-inestabilidad implícitos en interacciones espontáneas de individuos en continua evolución, disipación, acumulación de experiencia y aprendizaje. Además, Anderson [35] atribuye a la toma de decisiones un carácter temporal por la naturaleza dinámica del conocimiento. La temporalidad cognitiva le resta relevancia a la imposición de comportamientos y a la estandarización de tareas porque el sistema se auto-organiza en forma colectiva con base en procesos de evolución, adaptación e influencias mutuas. En la misma dirección, Mitleton-Kelly [36], estima la autonomía de las 
decisiones como una construcción permanente generada por las emergencias. Por lo tanto, la organización se mantiene lejos del equilibrio y sus integrantes están en proceso de adaptación continua, gracias al intercambio y decantación de información endógena y exógena.

Los aportes de Holland, Gell Mann, Stacey y Mitleton identifican cinco factores esenciales en una gestión compleja: las emergencias, la auto-organización, la evolución, la co-evolución y la retroalimentación. Las emergencias producen nuevo orden y disipan las estructuras actuales [16]. En consecuencia, la evolución individual se convierte en co-evolución como resultado del aprendizaje colectivo. Según Holland (1998) y Prigogine \& Nicolis (1997), el carácter contingente de las interacciones limita las predicciones y genera inestabilidad en las organizaciones abiertas y en desequilibrio permanente. Estas contribuciones de los investigadores del SFI bosquejan principios para conducir las emergencias, el desorden, las tensiones, las contradicciones y las novedades (Figura 2) generadas por la evolución de la organización y la co-evolución de los individuos. Entretanto, las incertidumbres y las predicciones originan la negentropía y las estructuras disipativas. En forma complementaria, Richardson [56] y Holland [37], consideran la auto-organización como un orden transitorio con una inestabilidad inscrita en lo predecible-impredecible, donde la flexibilidad, la robustez y la innovación son cualidades inherentes a los sistemas abiertos.

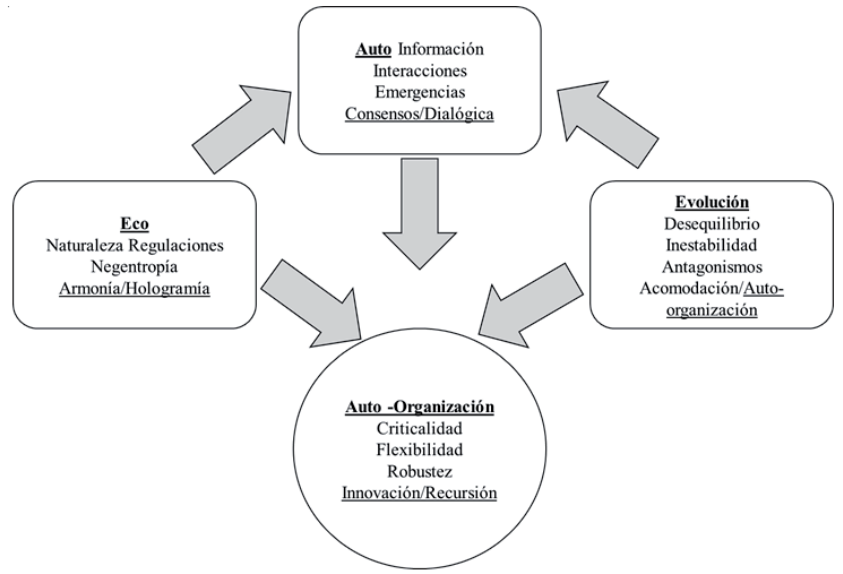

Figura 2. . Postulados para la gestión empresarial aportados por los Sistemas Complejos Fuente: Diseño a partir de las características de Holland [37] y Richardson [56], propiedades de Anderson [35], principios de Mitleton-Kelly [36] y los principios del pensamiento complejo de Morin [5].

En síntesis, el éxito de organizaciones orientadas por los sistemas complejos está relacionado con la dialógica permanente entre el orden - desorden y la capacidad de evolución, co-evolución y aprendizaje entre los integrantes de un sistema. El postulado principal para orientar una organización abierta y compleja, se fundamente en reconocer que el progreso de la organización depende más de las acciones colectivas de los trabajadores que de las acciones individuales de los directivos.

\section{Conclusiones}

Un nuevo marco teórico para las ciencias de la gestión cimentado en las ciencias de la complejidad, el pensamiento complejo y la auto-organización, presenta amplios avances a nivel nacional e internacional. En el ámbito de la ciencia contemporánea se analizan los estudios críticos de gestión (CMS), con inspiración anglo-sajona en la década de 1980; los sistemas adaptativos complejos (CAS) surgidos en Estados Unidos en la década de 1990; la corriente humanística de la gestión promovida por la Escuela Canadiense de Humanismo y Gestión (HEM) en la década de 1990. Actualmente, el grupo del Nuevo Pensamiento Administrativo de la Universidad del Valle en Colombia aboga por el rescate de la dignidad humana en la gestión y la necesidad de replantear la gestión lineal para disminuir la inequidad social y el deterioro progresivo del medio ambiente. La apuesta es evolucionar de la gestión lineal o clásica hacia la gestión post- estructuralista.

En particular, esta propuesta se origina en el pensamiento complejo de Edgar Morin, la contingencia estructural de Alfred Chandler, los estudios críticos de la gestión, los sistemas adaptativos complejos y el reconocimiento de las ciencias de la complejidad con un líder comprobado como Ilya Prigogine, Premio Nobel y creador de los principios relacionados con estructuras auto-organizadas en sistemas alejados del equilibrio. Las ciencias de la complejidad, integradas por la teoría del caos, la geometría de fractales, la dinámica del no equilibrio, la teoría de redes y la teoría de la catástrofe, le conceden a la corriente del pensamiento complejo el carácter universal de ciencia desde la década de 1980. Actualmente, en el contexto de la ciencia contemporánea, la mayor parte de las disciplinas tradicionales reciben influencia de los principios complejos, analógicos y contingentes. En esta nueva orientación de las ciencias es preciso admitir la influencia recibida del post-estructuralismo promovido por la corriente anglosajona liderada por Foucault, Adorno, Baudrillart, Horkheimer y Habermas, inspirados en Freud, Marx, Niesztche y Heidegger, entre el final del siglo XIX y comienzos del siglo XX. Así como la contribución del enfoque sistémico de Wiener y Bertalanffy en las décadas de 1940 y 1950.

Con la corriente compleja, la diferenciación tradicional entre ciencias humanas y ciencias básicas tiende a aminorarse; y se erige el desequilibrio fenomenológico que admite la preferencia por propuestas científicas susceptibles de contradicciones o refutaciones [57]. No obstante, la ciencia clásica o "normal", tomando la expresión de Kuhn [24], permanece aún displicente de la ciencia compleja. De esta forma, la soñada "era planetaria" de Morín, con postulados post-modernistas, aún no se han impregnado de armonía, convivencia, ciudadanía mundial y compromiso social. Aunque esta aspiración moriniana para algunos resulta ilusoria, la paulatina destrucción del planeta es un fuerte indicio del estado bárbaro de la incomprensión qué estamos viviendo [58]. 
Por su parte, en el marco de la biología y la ciencia contemporánea, los seres humanos como organizaciones vivientes desarrollan procesos auto-organizados asociados con la autopoiésis y el pensamiento complejo. Estos procesos se cimentan en las interacciones internas, la información proveniente del contexto y se vinculan con la autopoiésis porque propician acciones armoniosas entre los individuos y el entorno. En esta perspectiva, se reconoce la naturaleza abierta, exógena y entrópica que produce la vitalidad intrínseca de la auto-organización y que demanda una gestión armoniosa entre la naturaleza y la bio-antropología, congruente con mejor calidad de vida y equidad social, así como una disminución de la contaminación ambiental.

El pensamiento complejo, la computación, la cibernética y los sistemas apoyan los procesos inter-disciplinares, la profundización en investigaciones articuladas con objetivos comunes y facilitan el desarrollo de la trans-disciplinariedad. Así, se obvia el carácter disyuntivo de la investigación modernista. La ciencia contemporánea y las ciencias de la complejidad trascienden la disciplina y amplían el ámbito investigativo con la confluencia de factores históricos, sociales, físicos, químicos, psicológicos, políticos, económicos, filosóficos y culturales.

La corriente compleja de la gestión se inicia en instituciones de prestigio como la Escuela de Frankfurt en Alemania, la Escuela de Economía en Londres y la Escuela de Humanismo y Gestión de Canadá. Con la influencia de reconocidos pioneros como Morin, Braverman, Aktouf, Kaufman, Foerster, Horkheimer, Foucault, Le Moigne, entre otros. Colombia presenta significativos avances en el desarrollo de los estudios críticos de la gestión con el grupo de Perdurabilidad Empresarial de la Universidad del Rosario y la Escuela del Nuevo Pensamiento Administrativo de la Universidad del Valle. En particular se distingue el trabajo inspirador de Omar Aktouf, así como los escritos de Saavedra, Maldonado, Cruz y Sanabria, entre otros. Esta corriente se despliega a través de foros, publicaciones y contenidos curriculares en los niveles de pregrado y postgrado. La Universidad Nacional a través de la Facultad de Ciencias Económicas y la Escuela de Administración y Contaduría invitó a tres connotados conferencistas: Humberto Maturana, Fernando Cruz Konfly y Omar Aktouf, entre el segundo semestre de 2014 y primer semestre de 2015. Así se aporta a la construcción de una nueva epistemología para la gestión en el país, en particular para los programas de administración y gestión.

En la Universidad Nacional de Colombia [59], una de las instituciones líderes de la educación superior en el país y en Latinoamérica, operan diez (10) Centros de Pensamiento para "contribuir a solucionar los problemas de la sociedad", entre los cuales está el déficit comercial, reconocido como uno de los problemas estructurales del país causado por la escasa investigación científica. Porque importamos la mayor parte de los bienes de capital y exportamos los recursos naturales energéti- cos, mineros y agrícolas. Los datos son elocuentes, el déficit comercial alcanzó la cifra sin precedentes de US\$ 6.293 millones [60]. Surge la evidente propuesta de crear un centro de pensamiento para la gestión administrativa con una perspectiva compleja y post - estructuralista y con el propósito de generar creatividad e innovación en la solución de los problemas estructurales del desarrollo empresarial y económico colombiano. En especial con el compromiso de renovar la gestión instrumental enseñada en las universidades a través de manuales de funciones y procedimientos estandarizados, evaluaciones financieras centradas en el utilitarismo y falseadas por la exclusión de los costos ambientales, el mercadeo para el consumismo, el proceso administrativo burocratizado y la planeación estratégica taxativa. Para citar algunos temas aún enseñados en las universidades colombianas desde la fundación de los primeros programas de administración en la década de 1960 y bajo los principios clásicos de Taylor, Fayol, Ford, Webber y Mayo, entre otros.

A la gestión lineal o clásica se le atribuye gran influencia en la degradación e instrumentalización del individuo según las críticas preliminares de Braverman en la década de 1970, relativas a la descualificación del trabajo. Actualmente, esta postura es seguida por Aktouf con énfasis en las prácticas inhumanas en el trabajo. Adicionalmente, Le Moigne alude al excesivo positivismo, a la casuística y a la falta de epistemologías propias para fundamentar las ciencias de la gestión. Mientras que Simón cuestiona la fascinación de la gestión por las matemáticas y las finanzas a expensas de la desatención de la complejidad del individuo. Horkheimer plantea el desconocimiento de la razón en favor de la instrumentalización. Habermas señala la carencia de una verdadera participación democrática. Foucault compara la gerencia con un panóptico carcelario por el excesivo control. Saavedra califica al instrumentalismo como el lado oscuro de la gestión. Y Cruz Konfly aboga por la necesidad de rescatar la dignidad humana en la gestión. Igualmente, la gestión lineal se implica con la inequidad social, el deterioro progresivo del medio ambiente y la pérdida de la calidad de vida. Como causa sociológica primaria aparece la alta concentración de la riqueza y el predominio de la distribución de los beneficios del trabajo en favor de quienes poseen los recursos de inversión, con la consecuente concentración de la riqueza o crematística, en detrimento del bien vivir juntos, en palabras de Omar Aktouf [61]. Luego, una empresa sostenible en el tiempo debe velar, no solamente por sus intereses económicos, sino también por sus responsabilidades con el factor humano, la sociedad misma y el cuidado del ambiente [62].

Con inspiración de escuelas anglosajonas, francesas, norteamericanas, latinoamericanas y en el marco de las ciencias de la complejidad surgen nuevos conceptos para explicar la variabilidad crítica de los fenómenos humanos, sociales, físicos y económicos desde la segunda mitad del siglo XX. Así se configura un nuevo glosario para la gestión: emergencias, turbulencias, bifurcaciones, incertidumbres, disipación, robustez, innovación, inestabilidad, auto organización, evolución, 
co-evolución, maleabilidad y analogías. Este nuevo lenguaje configura el cambio epistemológico de la gestión. En consecuencia, el marco conceptual de la gestión clásica colmado de expresiones ingenieriles, psicológicas y sociológicas clásicas es reformado por un nuevo glosario proveniente de la biología, la física y las nuevas corrientes filosóficas y sociológicas. Se integra la simplicidad con la complejidad y las ciencias básicas con las ciencias humanas, en torno a una epistemología interdisciplinar e incluyente. Además, se reconoce la gran paradoja de la ciencia contemporánea: la verdad relativizada por la disipación, la temporalidad y la contingencia.

En forma puntual, se admite una nueva epistemología para respaldar el discurso académico de la innovación y la creatividad de la gestión, así como para revalidar el carácter ecléctico de la disciplina administrativa. Se trata de actualizar las ciencias de la gestión con base en las ciencias de la complejidad, la auto-organización, los sistemas adaptativos complejos (CAS), la cibernética, los sistemas generales, los principios del pensamiento complejo, los fundamentos de la complejidad y simplicidad; la dialógica orden - desorden, la auto organización, la evolución, la co-evolución, las emergencias, la flexibilidad y la robustez. Bienvenidos los nuevos cimientos epistemológicos de la gestión para respaldar el discurso académico de una verdadera innovación y creatividad. Emulando el estilo sugerente de Le Moigne, los actuales académicos de la gestión deberían incluir la corriente compleja en los programas y currículos con los postulados de Braverman, Foucault, Horkheimer, Habermás, Morin, Deming, Ouchi, Kauffman, Von Bertalanffy, Foerster, Aktouf, Cruz Konfly, Saavedra y Maldonado, entre otros. Para actualizar los contenidos de los programas de gestión, complementarlos y confrontarlos, en una simbiosis evolutiva, con los postulados de los autores de la corriente clásica y estructuralista como Taylor, Fayol, Mayo, Weber, Etzioni, Barnard y Drucker.

Con estos cambios estructurales y epistemológicos se pretende transformar la gestión instrumentalista y resultadista en una gestión robusta e innovadora con propósitos de estabilidad, crecimiento y distribución más equitativa de beneficios en función de la calidad de vida. Es la rebelión de las ciencias de la gestión frente a la imposición de modelos del mercado. Es la adopción de un liderazgo proactivo apoyado en los estudios críticos de la gestión iniciados hace más de cuatro décadas. Se evidencia un volumen crítico de teorías y experiencias que sustentan un nuevo marco conceptual con la aplicación de la complejidad, la auto-organización, la teoría crítica y el post-estructuralismo, pilares en la construcción de una nueva era para las ciencias de la gestión y en el despegue de una industria de bienes de consumo hacia una industria de bienes de capital apoyada en una gestión genuina colombiana.

"Nada parece más libre que un pájaro en el cielo. Nada es más autóctono que su vuelo. Y sin embargo, tal libertad, tal autonomía evidentes a primera vista, se descomponen en un segundo momento: el conocimiento que descubre los determinismos ecológicos, moleculares y genéticos a los cuales obedece el vuelo triunfante del pájaro".

\section{Agradecimientos}

Los autores estienden su agradecimiento a los señores Jairo Alexander Hernández Valero, por su apoyo como asesor metodológico e Ivonne Tatiana Hernández Valero por su apoyo en el diálogo de ideas.

\section{Referencias}

[1] C. E. Maldonado, "Complejidad de los sistemas sociales: un reto para las ciencias sociales," Cinta Moebio, no. 36, pp. 146-157, 2009.

[2] J. Von Neumann, Teoría de los autómatas reproductores. Illinois: Press Champaing, 1966.

[3] M. Delgado Ruíz, "Dinámicas identitarias y espacios públicos," Afers Int., no. 43-44, pp. 17-33, 1998.

[4] H. Atlan, "On a formal definition of organization," J. Theor. Biol., vol. 45, no. 2, pp. 295-304, 1974.

[5] E. Morin, Introducción al pensamiento complejo. Barcelona: Gedisa, 1998.

[6] H. . Maturana, F. . Varela, and R. Uribe, "Autopoiésis: The organization of living systems, its characterization and a model,” Biosystems, vol. 5, no. 4, pp. 187-196, 1974.

[7] E. Morin, El Método II: La Vida de la Vida. Madrid: Ediciones Cátedra, 1993.

[8] F. Hayek, Los orígenes de la libertad, la propiedad y la justicia. La Fatal Arrogancia. Madrid: Unión Editorial, 1990.

[9] H. Von Foerster, "Principios de auto-organización en un contexto socio-empresarial," Springer Berlin Heidelb., pp. 2-24, 1984.

[10] M. Crozier, El fenómeno burocrático. Buenos Aires: Amorrortu, 1969.

[11] I. Chiavenatto, Introducción a la Teoría General de la Administración. México: MacGraw Hill, 2011.

[12] E. Morin, Ciencia con consciencia. Barcelona: Anthropos, 1984.

[13] T. Déry, El ajuste de cuentas. México: Universidad Veracruzana, 2007.

[14] C. Maldonado, El mundo de las ciencias de la complejidad: Una investigación sobre qué son, su desarrollo y sus posibilidades. Bogotá: Universidad del Rosario, 2011. 
[15] H. . Maturana and F. . Varela, De máquinas y seres vivos. Autopoiesis: La organización de lo vivo. Buenos Aires: Lumen, 1994.

[16] S. Kauffman, At Home in the Universe: The Search for the Laws of Self-Organization and Complexity. New York: Oxford University Press, 1995.

[17] F. Hayek, Rules and Order. London: University Press, 1973.

[18] I. Prigonine, Tan sólo una ilusión: Una explotación del orden a partir del desorden. Barcelona: Tusquéts, 1983.

[19] I. Prigonine and G. Nicolis, Self-Organization in Non-Equilibrium Systems. New York: Wiley-Interscience, 1997.

[20] M. Gell - Mann, "Complex adaptative systems," in Complexity: methaphors, models, and reality, G. Cowan, D. Meltzer, and D. Pines, Eds. México: Adisson-Wesley, 1994, pp. 17-45.

[21] J. L. Le Moigne, "La incoherencia epistemológica de las ciencias de la gestión," Cuad. Econ., vol. 16, no. 26, pp. 163-185, 1997.

[22] D. Najmanovich, "Pensar la subjetividad. Complejidad, vínculos y emergencia," Utopía y Prax. Latinoam., vol. 6, no. 14, pp. 106-111, 2001.

[23] L. E. Bohorquez Arévalo, "La organización empresarial como sistema adaptativo complejo," Estud. Gerenciales, vol. 29, no. 127, pp. 258-265, 2013.

[24] T. Kuhn, La estructura de las revoluciones científicas. México: Fondo de cultura económica, 2006.

[25] L. Von Bertalanffy, "The theory of open systems in physics and biology," Science (80-. )., vol. 111, no. 2872, pp. 23-29, 1947.

[26] S. Kauffman, Investigaciones: complejidad, auto organización y nuevas leyes para una biología general. Barcelona: Tusquéts, 2003.

[27] R. D. Stacey, "The science of complexity: An alternative perspective for strategic changing processes," Strateg. Manag. J., vol. 16, no. 6, pp. 477-495, 1995.

[28] G. W. Leibniz and F. D. P. Samaranch, La profesión de fe del filósofo. Madrid: Aguilar, 1966.

[29] B. Nicolescu, Manifiesto de la transdisciplinariedad. New York: Suny Press, 2002.
[30] J. Thompson, Interdisciplinarity: history, theory and practice. Detroit: Wayne State University, 1990.

[31] M. Horkheimer, "Teoría crítica," Rev. Sociol., pp. 171-176, 1974.

[32] H. Braverman, Trabajo y capital monopolista: La degradación del trabajo en el siglo XX. México: Nuestro Tiempo, 1998.

[33] M. Foucault, Vigilar y castigar. El nacimiento de la prisión. Madrid: Siglo XXI, 2005.

[34] J. Habermás, Teoría de la acción comunicativa. Madrid: Taurus, 1987.

[35] P. Anderson, "Complexity theory and organization science,” Organ. Sci., vol. 10, no. 3, pp. 216-232, 1999.

[36] E. Mitleton-Kelly, The principles of complexity and enhabling infraestructures. In complex systems an evolucionary perspectives of organization. London: London School of Economics (LSD), 2003.

[37] J. H. Holland, Hidden order: How adaptation builds complexity. New York: Perseus Books Group, 1995.

[38] L. Von Bertalanffy, "An outline of general system theory,” Br. J. Philos. Sci., vol. 1, no. 2, pp. 134-165, 1950.

[39] N. Wiener, Cybernetics or Control and Comunication in Animal and the Machine. York: Monthly Review Press, 1965.

[40] E. Lorenz and D. Ruelle, The essence of chaos. Seatle: University of Washington Press, 1993.

[41] B. Mandelbrot, Los objetos fractales. Barcelona: Tusquéts, 1997.

[42] E. C. Zeeman, La teoría de catástrofes: Los trabajos seleccionados, 1972-1977. Reading: Addison-Wesley Publishing Co, 1977.

[43] A.-L. Barabasi, How everything is connected to everything else and what it means for business, science and everyday life. Cambridge: Plume, 2003.

[44] P. Allen, S. Maguiere, and B. McKelvey, Eds., The Sage Handbook of Complexity and Management. London: Sage, 2001.

[45] I. Prigonine, From Being to Becoming. Time and Complexity in the Physical Sciences. San Francisco: W. H. Freeman \& Co, 1980.

[46] J. H. Holland, Emergence from chaos to order. New York: 
Perseus Books Groups, 1998.

[47] R. Descartes, El discurso del método. Bogotá: Panamericana, 1999.

[48] H. A. Simon, "The New Science of Management Decision," Harvard Business Review, vol. 39. p. 104, 1961.

[49] J. M. Panea Márquez, Quérer la utopía: Razón y auto conservación en la Escuela de Frankfurt: una crítica de la razón instrumental. Sevilla: Universidad de Sevilla, 1996.

[50] C. J. Fernández Rodríguez, Vigilar y organizar: una introducción a los Critical Managment Studies. Madrid: Siglo XXI, 2007.

[51] F. Cruz Kronfly, "Pensamiento, obsesión por la verdad y gestión: legitimidad de los estudios críticos organizacionales," in Estudios críticos de la organización, R. Carvajal Baeza, Ed. Cali: Universidad del Valle, 2013, pp. 129-145.

[52] J. J. Saavedra Mayorga, "Descubriendo el lado oscuro de la gestión: Los Critical Management Studies," Investig. y Reflexión, vol. 17, no. 2, pp. 45-60, 2009.

[53] F. Cruz Kronfly, "Pensamiento, obsesión por la verdad y gestión: legitimidad de los estudios críticos organizacionales," in Conferencia llevada a cabo en la Escuela de Administración y Contaduría de la Universidad Nacional, Bogotá, Colombia.

[54] M. Bunge, Emergence and convergence: qualitative novelty and the unity of knowledg. Toronto: University of Toronto Press, 2003.

[55] H. . Maturana, "Cibernética en tiempo cero. Ceremonia de lanzamiento en Latinoamérica del XVI congreso de la organización mundial de sistemas y cibernética," in Conferencia llevada a cabo en la Escuela de Administración y Contaduría de la Universidad Nacional, Bogotá, Colombia.

[56] K. A. Richardson, "Managing complex organizations: complexity thinking and the science and art of management," Emerg. Complex. Organ., vol. 10, no. 2, pp. 13-26, 2008.

[57] K. R. Popper, La lógica de la investigación científica. Madrid: Tecnos, 1980.

[58] E. Morin, Los siete saberes necesarios para la educación del futuro. Paris: UNESCO, 1999.

[59] Universidad Nacional de Colombia, "Balance Social 2014," UN Periódico, p. 17, 2015.

[60] L. Sarmiento Anzola, "Muere el Sistema Nacional de Ciencia y Tecnología e Innovación," Desde Abajo, Bogotá, p. 2, 20-Apr-2015.
[61] O. Aktouf, “Teoría y práctica en el campo de la economía y la administración: un análisis crítico-humanista, histórico y epistemológico," in fundación de los programas de Administración y Contaduría de la Universidad Nacional, Bogotá, Colombia, 2015.

[62] J. A. Cortés, "El respeto, un valor que escas y castigar. El nacimiento de la prisión. Madrid: Siglo XXI, 2005.unity of knowledg. Toronto: University of Toronto Press, 2003. 